\title{
Wave-packet propagation in momentum space: Calculation of sharp-energy $S$-matrix elements
}

\author{
Zeki C. Kuruoğlu \\ Department of Chemistry, Bilkent University, 06533 Bilkent, Ankara, Turkey \\ F. S. Levin \\ Department of Physics, Brown University, Providence, Rhode Island 02912 \\ (Received 11 October 1991; revised manuscript received 26 March 1992)
}

\begin{abstract}
This paper examines momentum-space methods as a means of implementing a scattering-theoretic, long-time lemma on the extraction of sharp-energy $S$-matrix elements from a wave-packet description of collisions. In order to concentrate on the momentum space and computational aspects, the collision system studied is that of two particles; each has the mass of a nucleon. The formulation of the problem in momentum space avoids any spreading of the packets and allows for a straightforward analysis, which proceeds as follows. First, a time discretization is introduced, so that a conditionally stable, recursive, time-evolution scheme can be employed. The momentum dependence of the full wave packet is next expressed via an expansion in locally defined interpolating polynomials (here, piecewise quadratics), as in the finite-element method. Once the time evolution has progressed sufficiently, the $S$-matrix element $\widetilde{S}_{0}(q)$ can be extracted from the ratio of the $q$ th momentum components of the full and free wave packets. It is essential here that the numerically propagated free wave packet be used in this ratio, since otherwise numerical errors induced in the full wave packet are not canceled, and $\left|\widetilde{S}_{0}(q)\right|$ can become as large as 2 or more. Wave packets with central momenta $q_{0}$ equal to 1,2 , and $4 \mathrm{fm}^{-1}$ (energies ranging from about 30 to $500 \mathrm{MeV}$ ) have been studied, and the behavior of the wave packets and $\widetilde{S}_{0}(q)$ for several time intervals, extraction times, numbers of mesh points, etc., have been explored. In general, results with errors of less than at most a few percent are easily obtainable.
\end{abstract}

PACS number(s): 03.65.Nk, 03.80. $+\mathrm{r}$

\section{INTRODUCTION}

The most fundamental description of quantal scattering is via wave packets and the time-dependent Schrödinger equation. The standard result of using such a description has been a set of averaged scattering parameters, e.g., average $S$-matrix elements or phase shifts, where the average is over the averaging or profile function used to define the initial wave packet [1].

This description, however, is not the one generally followed in analyses of scattering experiments. Instead, a time-independent analysis involving sharp (nonnormalizable) momentum states is used $[1,2]$. This analysis is employed because one is normally interested in the scattering parameters evaluated at sharp values of momentum or energy. The time-independent (TI) description can be obtained from the time-dependent (TD) one by taking the wave-packet averaging function to be a $\delta$ function in momentum [1]. An alternative derivation of the TI equations makes use of an averaging function which is made very narrow [1]; variations away from the central value are then argued to be negligible.

However, it is not necessary to make the transition from the TD to the TI description in order to obtain sharp values of $S$-matrix elements; they can also be extracted (to numerical accuracy) from a wave-packet description by making use of a long-time scatteringtheoretic lemma [1]. It states that for sufficiently long times, sharp $S$-matrix elements can be extracted from a wave-packet analysis, thus producing results independent of the form of the averaging function defining the packet.
Application of this lemma has been pioneered by workers in the chemical and molecular physics community, who used it to study atom-diatom collisions below the threshold for dissociation or breakup into three fragments (atoms or ions) [3,4].

More recently, we have successfully applied the longtime lemma in the three-particle nuclear case at bombarding energies well above the breakup threshold [5]. In the three-particle system at these energies, both two-body rearrangement and three-body breakup can occur. Just as in the TI approach, the analysis of such processes within the TD framework is complicated [5,6]. These complications tend to obscure somewhat those arising from use of the TD description itself. For example, one of the concerns is how to avoid boundary reflection problems that adversely affect the computational efficiency of coordinate-space methods, especially with respect to collisions requiring long propagation times. Wave-packet propagation in the interaction picture [7] and timedependent integral-equation methods [8] are two examples of recent efforts exploring alternatives to the conventional Schrödinger-picture, coordinate-space wave-packet propagation.

In this article, we describe a Schrödinger-picture momentum-space wave-packet method which, when used in conjunction with a computational version of the longtime lemma, appears to be well suited for problems requiring long propagation times. A major advantage of the method derives from the nonmoving and nonspreading nature of wave packets in momentum space. In the present paper, the method is applied to a spinless two- 
particle system, which, being free of the complications arising from rearrangement scattering and breakup, allows us to concentrate on the numerical aspects and the problems of extracting sharp-energy $S$-matrix elements from the wave-packet description. Our analysis carries over to the three-particle collision system, which we plan to report on elsewhere, the emphasis in this latter report being on the rearrangement and breakup aspects: the present analysis of the two-particle problems provides the background against which the three-particle calculations were carried out.

The organization of this paper is as follows. In the following section a heuristic derivation of the long-time lem$\mathrm{ma}$ is given. Section III is concerned with the solution procedures, including discussions of the time and space discretizations and of the time-evolution algorithm used. The details for implementing the solution procedures are the subject of Sec. IV, while numerical results are covered in Sec. V.

\section{LONG-TIME LEMMA}

In this section, we introduce notation and give a heuristic derivation of the long-time lemma. Since only the relative motion part of the spinless two-particle system need be considered, we are dealing with an effective one-body problem, the Hamiltonian for which is

$$
H=H_{0}+V \text {. }
$$

Here $H_{0}$ is the relative motion kinetic-energy operator and $V$ is the interparticle potential. We take $V$ to be spherically symmetric and of short range. The relative coordinate is denoted by $\mathbf{r}$ and the conjugate momentum by $q$. q):

The eigenstates of $H_{0}$ are the sharp-momentum states

$$
H_{0}|\mathbf{q}\rangle=E_{q} \mid \mathbf{q},
$$

where $E_{q}=q^{2} / 2 \mu$, with $\mu$ being the reduced mass (we are employing units in which $\hbar=1)$. The momentum states are normalized as $\left\langle\mathbf{q} \mid \mathbf{q}^{\prime}\right\rangle=\delta\left(\mathbf{q}-\mathbf{q}^{\prime}\right)$. Adopting a similar $\delta$-function normalization for the position states $|\mathbf{r}\rangle$, we have $\langle\mathbf{r} \mid \mathbf{q}\rangle=e^{i \mathbf{q} \cdot \mathbf{r}} /(2 \pi)^{3 / 2}$.

Let the precollision state of the system at the "distant past" $t_{P}$ be described by an incoming wave-packet state $\left|\Phi_{\mathrm{q}_{0}}\left(t_{P}\right)\right\rangle$ :

$$
\left|\Phi_{\mathbf{q}_{0}}\left(t_{P}\right)\right\rangle=\int d \mathbf{q}|\mathbf{q}\rangle f_{\mathrm{q}_{0}}(\mathbf{q})
$$

where $f_{\mathbf{q}_{0}}(\mathbf{q})$ is the averaging (or profile) function, with $\mathbf{q}_{0}$ being the central or average momentum. We assume that the coordinate-space amplitude $\left\langle\mathbf{r} \mid \Phi_{\mathrm{q}_{0}}\left(t_{P}\right)\right\rangle$ is negligibly small within the range of the potential. In the absence of the potential, the system evolves according to $\left|\Phi_{\mathrm{q}_{0}}(t)\right\rangle=e^{-i H_{0}\left(t-t_{P}\right)}\left|\Phi_{\mathrm{q}_{0}}\left(t_{P}\right)\right\rangle$. The momentum-space representation of the in state $\left|\Phi_{\mathrm{q}_{0}}(t)\right\rangle$ reads

$$
\begin{aligned}
\left\langle\mathbf{q} \mid \Phi_{\mathbf{q}_{0}}(t)\right\rangle & =e^{-i E_{q}\left(t-t_{P}\right)}\left\langle\mathbf{q} \mid \Phi_{\mathbf{q}_{0}}\left(t_{P}\right)\right\rangle \\
& =e^{-i E_{q}\left(t-t_{P}\right)} f_{\mathrm{q}_{0}}(\mathbf{q}) .
\end{aligned}
$$

Note that the momentum probability density of the free wave packet is independent of time, and, hence, the momentum support of the in state is constant. This is an important advantage of the momentum-space wavepacket method.

The full scattering state $\left|\Psi_{\mathrm{q}_{0}}(t)\right\rangle$ is the solution of the time-dependent Schrödinger equation (TDSE),

$$
i \frac{\partial}{\partial t}\left|\Psi_{\mathrm{q}_{0}}(t)\right\rangle=H\left|\Psi_{\mathrm{q}_{0}}(t)\right\rangle,
$$

subject to the initial condition

$$
\left|\Psi_{\mathrm{q}_{0}}\left(t_{P}\right)\right\rangle=\left|\Phi_{\mathrm{q}_{0}}\left(t_{P}\right)\right\rangle \text {. }
$$

To be formally rigorous, the initial condition must be formulated as a strong limit in the infinite past [1], viz.,

$$
\lim _{t \rightarrow-\infty} \|[|\Psi(t)\rangle-|\Phi(t)\rangle] \|=0 \text {. }
$$

However, the replacement in practice of the infinite-past limit with the distant, but finite, past condition (6) can be justified if the potential $V(r)$ can be neglected beyond a finite distance $r_{V}$ and $\left\langle\mathbf{r} \mid \Phi_{\mathrm{q}_{0}}\left(t_{P}\right)\right\rangle$ has negligible amplitude within $0 \leq r \leq r_{V}$.

As the collision develops in time, the system evolves into an asymptotically free (noninteracting) configuration, the out state. Let $t_{F}$ be defined by the condition

$$
\int_{0}^{r_{V}} r^{2} d r \int d \Omega_{r}\left|\left\langle\mathbf{r} \mid \Psi_{\mathrm{q}_{0}}(t)\right\rangle\right|^{2} \approx 0, \quad t \geq t_{F} .
$$

That is, the wave packet emerges at $t_{F}$ from the interaction region, and the further time evolution of the wave packet is governed by $H_{0}$. The $S$ operator $\hat{S}$ is then defined as [1]

$$
\left|\Psi_{\mathrm{q}_{0}}(t)\right\rangle=\hat{S}\left|\Phi_{\mathrm{q}_{0}}(t)\right\rangle, \quad t \geq t_{F} .
$$

How large $t_{F}$ must be for (8) to be valid depends on $q_{0}$, the range of $V$, and the shape of the initial wave packet as well as on the accuracy one demands in any particular computation. Of course, the formal theory would require taking the $t \rightarrow \infty$ limit.

We can remove the restriction $t \geq t_{F}$ from (8) by defining for all $t$ the out state $\left|\chi_{\mathrm{q}_{0}}(t)\right\rangle$ via

$$
\left|\chi_{\mathrm{q}_{0}}(t)\right\rangle=e^{-i H_{0}\left(t-t_{F}\right)}\left|\Psi_{\mathrm{q}_{0}}\left(t_{F}\right)\right\rangle,
$$

which implies, for $t<t_{F}$, a backpropagation of $\left|\chi_{\mathrm{q}_{0}}\left(t_{F}\right)\right\rangle$ under $H_{0}$. The out state $\left|\chi_{\mathbf{q}_{0}}(t)\right\rangle$ can then be interpreted as the free wave packet which evolves into $\left|\Psi_{\mathrm{q}_{0}}(t)\right\rangle$ in the asymptotic future $t \geq t_{F}$. We can then rewrite Eq. (8) as

$$
\left|\chi_{\mathbf{q}_{0}}(t)\right\rangle=\hat{S}\left|\Phi_{\mathbf{q}_{0}}(t)\right\rangle \text {. }
$$

In the formal theory, as a result of the so-called intertwining property of the wave operators [1], Eq. (10) holds for all $t$. In its computational implementation, however, it is subject to two restrictions.

(i) The replacement of the full Hilbert space of the system with a finite approximation space implies either ex- 
plicitly or implicitly a finite computational region in the coordinate space. Denoting by $t_{\max }\left(t_{\text {min }}\right)$ the time after (prior to) which the in and out states begin to be reflected from the boundaries of the computational coordinate domain, then Eqs. (9) and (10) will be valid only in the interval $\left[t_{\min }, t_{\max }\right]$.

(ii) Since the infinite-past and infinite-future limits are approximated, the $S$ operator extracted from Eq. (10) will show some $t$ dependence. The stability of $S$ against $t$ in a given calculation serves as a measure of the adequacy of the computational procedures.

The average $S$-matrix $\langle S\rangle$ may now be obtained via $\langle S\rangle=\left\langle\Phi_{\mathrm{q}_{0}}(t)|\hat{S}| \Phi_{\mathrm{q}_{0}}(t)\right\rangle=\left\langle\Phi_{\mathrm{q}_{0}}(t) \mid \chi_{\mathrm{q}_{0}}(t)\right\rangle$. Unless the initial wave packet has a very narrow momentum distribution about $\mathrm{q}_{0},\langle S\rangle$ will in general be different from $\left\langle\mathbf{q}_{0}|S| \mathbf{q}_{0}\right\rangle$. Our goal is to eliminate the effect of the averaging function $f_{\mathrm{q}_{0}}(\mathbf{q})$ and obtain sharp-energy $S$ matrix elements in place of the averages $\langle S\rangle$. To do so, we project both sides of (10) onto the momentum state $\langle\mathbf{q}|$, and obtain

$$
\begin{aligned}
\left\langle\mathbf{q} \mid \chi_{\mathbf{q}_{0}}(t)\right\rangle & =\left\langle\mathbf{q}|\hat{S}| \Phi_{\mathbf{q}_{0}}(t)\right\rangle \\
& =\int d \mathbf{q}^{\prime}\left\langle\mathbf{q}|\hat{S}| q^{\prime} \mid \Phi_{\mathbf{q}_{0}}(t)\right\rangle .
\end{aligned}
$$

Since $\hat{S}$ is energy conserving, we have [1]

$$
\left\langle\mathbf{q}|\hat{S}| \mathbf{q}^{\prime}\right\rangle=\left\langle q \Omega_{q}|S| q \Omega_{q^{\prime}}\right\rangle \delta\left(E_{q}-E_{q^{\prime}}\right),
$$

where $S$ is the reduced scattering operator, and $\Omega_{q}$ denotes the unit vector along q. Hence Eq. (10) becomes

$\left\langle\mathbf{q} \mid \chi_{\mathbf{q}_{0}}(t)\right\rangle=\mu q \int d \Omega_{q^{\prime}}\left\langle q \Omega_{q}|S| q \Omega_{q^{\prime}}\right\rangle\left\langle q \Omega_{q^{\prime}} \mid \Phi_{\mathrm{q}_{0}}(t)\right\rangle$.

Of course, Eq. (13) does not yet allow us to compute $\left\langle q \Omega|S| q \Omega^{\prime}\right\rangle$ from a single wave-packet calculation. It is possible in principle to discretize the angular integral in (13) by an $N_{\Omega}$-point quadrature rule, and to apply (13) to $N_{\Omega}$ different wave packets $\Psi_{\mathrm{q}_{0 i}}$ obtained from $N_{\Omega}$ linearly independent initial wave packets $\Phi_{\mathrm{q}_{0 i}}, i=1,2, \ldots, N_{\Omega}$. However, apart from being computationally costly, this procedure is prone to instabilities, for it would be difficult to guarantee the same degree of accuracy for each point of the angular quadrature mesh with initial packets of differing angular dependence.

A more practical method of handling the angular dependence in Eq. (13) is to use the rotational invariance of the potential. For this purpose, let us introduce the partial-wave momentum states $|q l m\rangle$ via

$$
|q l m\rangle=\int d \Omega_{q} Y_{\lim }^{*}\left(\Omega_{q}\right)|\mathbf{q}\rangle,
$$

so that $|\mathbf{q}\rangle=\sum_{l, m}|q l m\rangle Y_{l m}\left(\Omega_{q}\right)$. The partial-wave position states $|r l m\rangle$ are similarly defined. The normalization convention is such that $\left\langle q l m \mid q^{\prime} l^{\prime} m^{\prime}\right\rangle$ $=\delta_{l l^{\prime}} \delta_{m m^{\prime}} \delta\left(q-q^{\prime}\right) / q^{2}, \quad$ and $\quad\langle\mathbf{r} \mid q l m\rangle$ $=\sqrt{2 / \pi} i^{l} Y_{l m}\left(\Omega_{r}\right) j_{l}(q r)$, with $j_{l}$ denoting the spherical Bessel function. Any rotationally invariant operator $A$ can be expanded as

$$
\left\langle\mathbf{q}|A| \mathbf{q}^{\prime}\right\rangle=\sum_{l, m} Y_{l m}^{*}\left(\Omega_{q}\right) Y_{l m}\left(\Omega_{q^{\prime}}\right) A_{l}\left(q, q^{\prime}\right)
$$

where $A_{1}\left(q, q^{\prime}\right)$ ( $\left.\equiv\langle q \operatorname{lm}|A| q \operatorname{lm}\rangle\right)$ is independent of $m$. For the scattering operator, we have $\hat{S}_{l}\left(q, q^{\prime}\right)$ $=\delta\left(E_{q}-E_{q^{\prime}}\right) S_{l}(q), \quad$ where $\quad S_{l}(q) \equiv \mu q\langle q \operatorname{lm}|S| q \operatorname{lm}\rangle$ $=e^{2 i \delta_{l}^{q}(q)}$, with $\delta_{l}$ being the phase shift.

By projecting (10) or (8) onto the partial-wave state $|q l m\rangle$, we obtain the partial-wave versions of the longtime lemma:

$$
S_{l}=\frac{\left\langle q \operatorname{lm} \mid \chi_{\mathrm{q}_{0}}(t)\right\rangle}{\left\langle q \operatorname{lm} \mid \Phi_{\mathrm{q}_{0}}(t)\right\rangle}
$$

or

$$
S_{l}=\frac{\left\langle q \operatorname{lm} \mid \Psi_{\mathrm{q}_{0}}(t)\right\rangle}{\left\langle q \operatorname{lm} \mid \Phi_{\mathrm{q}_{0}}(t)\right\rangle} .
$$

Although these two forms of the lemma are valid strictly in the limit $t \rightarrow \infty$, sufficient accuracy has been achieved for values of $t$ that are not only finite but also relatively small. In computational implementation, Eq. (16) is valid for $t_{\min } \leq t \leq t_{\max }$, while Eq. (17) is valid for $t_{F} \leq t \leq t_{\max }$. To numerically implement the above lemma, we can proceed in one of two ways.

(i) The full wave packet $\Psi_{\mathrm{q}_{0}}\left(\mathbf{q}, t_{F}\right)$ can be obtained by numerical solution of the TDSE directly in (the threedimensional) momentum space. Partial-wave $S$-matrix elements can then be obtained by projection onto angular momentum states.

(ii) Alternatively, the TDSE and the initial condition can be first projected on angular momentum states, and the resulting (decoupled) partial-wave TDSE's (in one space variable) can then be integrated for each partialwave separately.

The first procedure, in its most general form, does not make use of the rotational invariance, and can possibly be useful in cases where the partial-wave series is slowly converging. Of course, if the approximation space and the initial wave packet are characterized by definite angular momentum quantum numbers, then the two approaches become equivalent. Since the second approach is adopted in the present paper, we expand the wave packet as

$$
\left\langle\mathbf{q} \mid \Psi_{\mathbf{q}_{0}}\right\rangle=\sum_{l, m} Y_{l m}^{*}\left(\Omega_{q}\right) Y_{l m}\left(\Omega_{q_{0}}\right) \psi_{q_{0} l}(q, t) .
$$

The amplitudes $\left\langle\mathbf{q} \mid \Phi_{\mathbf{q}_{0}}(t)\right\rangle$ and $\left\langle\mathbf{q} \mid \chi_{\mathrm{q}_{0}}(t)\right\rangle$ and the profile function $f_{\mathrm{q}_{0}}(\mathbf{q})$ are similarly decomposed into partialwave components, denoted by $\phi_{q_{0} l}(q, t), \chi_{q_{0} l}(q, t)$, and $f_{q_{0} l}(q)$, respectively.

The partial-wave (PW) TDSE is

$i \frac{\partial}{\partial t} \psi_{q_{0} l}(q, t)=\frac{q^{2}}{2 \mu} \psi_{q_{0} l}(q, t)+\int q^{\prime 2} d q^{\prime} V_{l}\left(q, q^{\prime}\right) \psi_{q_{0} l}\left(q^{\prime}, t\right)$,

with the initial condition 


$$
\psi_{q_{0} l}\left(q, t_{P}\right)=\phi_{q_{0} l}\left(q, t_{P}\right) .
$$

Note that each initial partial-wave packet $\phi_{q_{0} l}\left(q, t_{P}\right)$ can be chosen separately, i.e., without having to refer to a full three-dimensional packet $\Phi_{\mathrm{q} 0}\left(\mathbf{q}, t_{P}\right)$.

The $S$ matrix in its long-time lemma formulation can now be evaluated via

$$
S_{l}(q)= \begin{cases}\frac{\psi_{q_{0} l}(q, t)}{\phi_{q_{0} l}(q, t)} & \text { for } t_{F} \leq t \leq t_{\max } \\ \frac{\chi_{q_{0} l} l}{\phi_{q_{0} l}(q, t)} & \text { for } t_{\min } \leq t \leq t_{\max }\end{cases}
$$

Since $\left|S_{l}\right|^{2}=1$, the momentum probability density of the out state must be the same as that of the in state. Scattering therefore manifests itself as a modification of the phase of the free wave packet.

The numerical time evolution of the full wave packet necessarily involves an approximation of the kineticenergy operator due to the space and time discretizations. Treating $H_{0}$ approximately (i.e., numerically) in the context of the full dynamics, but analytically with respect to free wave-packet evolution, gives rise to a consistency problem. As is often emphasized in a formal scattering theory context, the $S$ matrix represents a quantitative comparison of dynamics for two Hamiltonians, namely $H_{0}+V$ and $H_{0}$. Therefore it is essential that $H_{0}$ be treated at the same level of approximation in both contexts. Accordingly, we use the numerically propagated free wave packet in the computational version of the longtime lemma. Failure to do so leads to nonunitary values of $S_{l}(q)$, as demonstrated in Sec. V.

\section{SOLUTION PROCEDURES}

In order to determinate $S_{l}(q)$ computationally, discretizations in time and space must be introduced. Wave packets do not move or spread in momentum space: their support remains constant. Because of this, our computations were carried out in momentum space, even though the potentials become integral operators, a feature easily handled numerically in general.

\section{A. Time discretization}

Time discretization is used to integrate the PW TDSE numerically. Of the various procedures available to accomplish this, we chose the conditionally stable, central difference method [9]. This leads to a recursion relation for affecting the time evolution. The method involves dividing the time interval into a mesh of spacing $\delta t$, so that $t_{j}=t_{P}+j \delta t$. Equation (19), which is first order in time, becomes

$$
\left|\psi_{q_{0} l}\left(t_{j+1}\right)\right\rangle \approx\left|\psi_{q_{0} l}\left(t_{j-1}\right)\right\rangle-2 i \delta t H_{l} \mid \psi_{q_{0} l}\left(t_{)}\right\rangle,
$$

where $H_{l}$ is the partial-wave Hamiltonian, given in momentum space by $H_{l}\left(q, q^{\prime}\right)=q^{2}(2 \mu)^{-1} \delta\left(q-q^{\prime}\right)$ $+V_{l}\left(q, q^{\prime}\right)$.

Equation (23) is well suited to implementation on vec- tor processors since the basic steps are repeated matrixvector multiplications and vector-vector additions. Although more sophisticated algorithms are available $[10,11]$, time propagation was never a problem in carrying out our calculations.

\section{B. Spatial discretization}

To realize the numerical solution of the PW TDSE, the partial-wave Hilbert space has to be approximated by a finite approximation space. The approximation space is spanned by a chosen set of basis states, and has to be large enough to allow an efficient approximation of $\left|\psi_{q_{0} l}(t)\right\rangle$ and $\left|\phi_{q_{0} l}(t)\right\rangle$ over a reasonably large time period. Denoting the basis states for the $l$ th partial wave by $\left|u_{n l}\right\rangle, n=1,2, \ldots, N$, their momentum representation is $u_{n l}(q)$. Rather than choosing to use a typical set of basis states defined globally, that is, over the entire momentum space, we employed instead a locally defined basis of piecewise interpolates, as in the finite-element method [11].

The first step in carrying out the momentum discretization is to reduce the range of momentum variables from the infinite real line $[0, \infty]$ to the finite domain $\left[0, q_{\max }\right]$. The cutoff $q_{\max }$ is mainly determined by the momentum support of the initial wave packet, although the large momentum behavior of $V_{l}\left(q, q^{\prime}\right)$ also plays a role. The finite momentum domain is next divided into a set of nonoverlapping subdomains (the "elements" of the finite-element method), and the piecewise interpolates are defined over this mesh. In our calculations the $u_{n l}(q)$ were taken to be piecewise quadratics, whose functional form is given in Sec. IV C.

The wave packets are expanded on the approximation space via

$$
\left|\widetilde{\psi}_{q_{0} l}(t)\right\rangle=\sum_{n}\left|u_{n l}\right\rangle C_{n l}(t)
$$

and

$$
\left|\tilde{\phi}_{q_{0} l}(t)\right\rangle=\sum_{n}\left|u_{n l}\right\rangle B_{n l}(t),
$$

where the $q_{0}$ dependence of the expansion coefficients is suppressed and the tilde denotes a numerically determined quantity.

When expansion (24) is used in the PW-TDSE, it will give rise to an error term

$$
|\mathscr{E}\rangle=\sum_{n}\left[i \frac{\partial}{\partial t}-H_{l}\right]\left|u_{n l}\right\rangle C_{n l}(t) .
$$

In the Galerkin method, the error $|\mathscr{E}\rangle$ is required to be orthogonal to the expansion functions $\left|u_{n}\right\rangle$, i.e., $\left\langle u_{n} \mid \mathscr{E}\right\rangle=0$. This requirement yields

$$
i \sum_{n^{\prime}} \Delta_{n n^{\prime}} \dot{C}_{n^{\prime}}(t)=H_{n n^{\prime}} C_{n^{\prime}}(t),
$$

where the partial-wave index has been suppressed for notational simplicity and

$$
\Delta_{n n^{\prime}}=\left\langle u_{n} \mid u_{n^{\prime}}\right\rangle=\int q^{2} d q u_{n^{\prime}}(q) u_{n}(q),
$$


while

$$
H_{n^{\prime} n}=\left\langle u_{n^{\prime}}\left|H_{l}\right| u_{n}\right\rangle .
$$

The overlap integrals $\Delta_{n^{\prime} n}$ will form a banded matrix of relatively narrow width, while the Hamiltonian matrix will essentially be full due to the nonlocal nature of the potential in the momentum representation.

In an obvious matrix notation, (27) reads $i \Delta \dot{\mathbf{C}}(t)=\mathbf{H C}(t)$. Using the central difference approximation for the time derivative, a recursive time propagation scheme is obtained:

$$
\mathbf{C}\left(t_{j+1}\right)=\mathbf{C}\left(t_{j-1}\right)-2 i \delta t \mathbf{\Delta}^{-1} \mathbf{H C}\left(t_{j}\right) .
$$

An alternative procedure to calculate the expansion coefficients is the collocation method. In this case, a set of $N$ points $\left\{q_{\alpha}\right\}_{\alpha=1}^{N}$ in the interval $\left[0, q_{\max }\right]$ is selected, and the error is required to vanish on this set of momentum points, viz., $\left\langle q_{\alpha} \mid \mathscr{E}\right\rangle=0$. This leads to

$$
\mathbf{C}\left(t_{j+1}\right)=\mathbf{C}\left(t_{j-1}\right)-2 i \delta t \bar{\Delta}^{-1} \overline{\mathbf{H}} \mathbf{C}\left(t_{j}\right),
$$

where

$$
\bar{\Delta}_{\alpha n}=\left\langle q_{\alpha} \mid u_{n}\right\rangle=u_{n}\left(q_{\alpha}\right)
$$

and

$$
\bar{H}_{\alpha n}=\left\langle q_{\alpha}\left|H_{l}\right| u_{n}\right\rangle .
$$

Since the basis functions are real, both schemes involve the inversion of a real matrix, which, however, has to be performed only once during all calculations using the same set of basis functions (and the same set of collocation points).

Setting $t_{P}=0$ in the rest of this article, the pair of coefficient vectors $\mathbf{C}(0)$ and $\mathbf{C}(\delta t)$ are needed to initiate the time propagation. By appropriate choices of the initial wave-packet parameters, the coordinate-space support of the incident wave packet at $t=0$ and $\delta t$ can be arranged to lie well outside the range of the potential. Under this assumption, we can set $C_{n}(0)=B_{n}(0)$ and $C_{m}(\delta t)=B_{m}(\delta t)$. The same assumption allows us to obtain $\left|\phi_{q_{0}}(\delta t)\right\rangle \quad$ analytically, viz., $\left\langle q \mid \phi_{q_{0}}(\delta t)\right\rangle$ $=e^{-i E_{q} \delta t} f_{q_{0} l}(q)$. Thus the expansion coefficients $B_{n}(0)$ and $B_{n}(\delta t)$ can be evaluated by applying either the Galerkin or the collocation criterion to Eq. (25):

$$
\left\langle w_{n} \mid \phi_{q_{0}}(t)\right\rangle=\sum_{n^{\prime}}\left\langle w_{n} \mid u_{n^{\prime}}\right\rangle B_{n^{\prime}}(t)
$$

where $\left|w_{n}\right\rangle=\left|u_{n}\right\rangle$ for the Galerkin method, and $\left|w_{n}\right\rangle=\left|q_{\alpha}\right\rangle$ for the collocation method.

Although the exact time evolution of the free wave packet is used for the first time step, we use the numerically propagated free wave packet in the extraction of the $S$ matrix via the long-time lemma, which basically compares the free and full wave packets. The numerical evolution procedure for the free wave packet is completely analogous to that for the full wave packet. The Galerkin method gives

$$
\mathbf{B}\left(t_{j+1}\right)=\mathbf{B}\left(t_{j-1}\right)-2 i \delta t \Delta^{-1} \mathbf{H}_{0} \mathbf{B}\left(t_{j}\right),
$$

while the collocation version reads

$$
\mathbf{B}\left(t_{j+1}\right)=\mathbf{B}\left(t_{j-1}\right)-2 i \delta t \bar{\Delta}^{-1} \overline{\mathbf{H}}_{0} \mathbf{B}\left(t_{j}\right),
$$

where $\left(\mathbf{H}_{0}\right)_{n^{\prime} n}=\left\langle u_{n^{\prime}}\left|H_{0}\right| u_{n}\right\rangle$ and $\left(\overline{\mathbf{H}}_{0}\right)_{n^{\prime} n}=\left\langle q_{n^{\prime}}\left|H_{0}\right| u_{n}\right\rangle$.

Note that the same mesh sizes must be used in the calculation of the free and full wave packets. Without doing so, the numerical errors that enter into the numerator and denominator of (10) or (17) do not cancel.

\section{IMPLEMENTATION}

\section{A. Two-particle interaction}

The interaction $V$ was taken to be of $S$-wave separable form:

$$
V_{0}\left(q, q^{\prime}\right)=\xi(q) \lambda \xi\left(q^{\prime}\right)
$$

with a Yamaguchi form factor [12]

$$
\xi(q)=1 /\left(q^{2}+\beta^{2}\right) .
$$

Here $\beta$ and $\lambda$ are the range and strength parameters, respectively. In our calculations, $\beta$ was chosen to be 1.444 $\mathrm{fm}^{-1}$, while $\lambda$ was selected to yield the bound-state energy $\varepsilon_{b}=-2.23 \mathrm{MeV}$. The two-body bound state simulates a deuteron. The analytic form of the $S$ matrix for this model is known [12].

\section{B. Incident wave packet}

Since each partial wave propagates independently of the others, we have limited ourselves to the $S$ wave $(l=0)$ case. This choice is sufficient for evaluating the formal and numerical procedures used herein. For the incident packet, the $S$-wave averaging (or profile) function has been chosen as an incoming Gaussian wave packet:

$$
\begin{aligned}
f_{q_{0}}(q)=\frac{A^{\prime}}{q}\left(e^{i\left(q-q_{0}\right) r_{0}} e^{-\left(q-q_{0}\right)^{2} d^{2} / 2}\right. & \\
& \left.+e^{i\left(q+q_{0}\right) r_{0}} e^{-\left(q+q_{0}\right)^{2} d^{2} / 2}\right),
\end{aligned}
$$

where $A^{\prime}$ is a normalization constant. Here we have suppressed the partial wave index $l=0$. The initial $S$ wave packet is then given as

$$
\left|\phi_{q_{0}}(t=0)\right\rangle=\int q^{2} d q|q\rangle f_{q_{0}}(q),
$$

where $|q\rangle$ denotes an $S$-wave momentum state. The coordinate representation of the initial wave packet is then

$$
\left\langle r \mid \phi_{q_{0}}(t=0)\right\rangle=A e^{-\left(r-r_{0}\right)^{2} / 2 d^{2}} \frac{e^{-i q_{0} r}}{r},
$$

with $A^{-2}=d \sqrt{\pi}$. It is evident from this expression that $r_{0}$ is the average position of the initial wave packet, while $d$ is its width. Furthermore, we see from (4) and (40) that

$$
\left\langle q \mid \phi_{q_{0}}(t)\right\rangle=e^{i q^{2} t / 2 \mu} f_{q_{0}}(q),
$$

i.e., in momentum space, the packet does not spread, as stated in the foregoing. Note also that $f_{q_{0}}(q)$ can be of 
any form: we have used a Gaussian for convenience only. Typical values used for $r_{0}$ and $d$ were $r_{0}=10 \mathrm{fm}$ and $d \approx 2 \mathrm{fm}$, respectively, while sampled values of $q_{0}$ were 1 , 2 , and $4 \mathrm{fm}^{-1}$.

\section{Interpolation basis}

The expansion basis we have used is a set of interpolating polynomials of piece-wise quadratic form. They are most easily described in terms of the momenta local to any one of the $I$ subintervals (or elements) which form a partition of the computational domain $\left[0, q_{\max }\right]$. The $p$ th partition is the interval $\left[Q_{p}, Q_{p+1}\right], p=1,2, \ldots, I$, whose midpoint is denoted $\bar{Q}_{p}$, as in Fig. 1 . Note that $Q_{1}=0$ and $Q_{I+1}=q_{\max }$. Not all intervals need be of the same length.

The interpolates are defined on the interval $[-1,1]$. We therefore introduce the mapping of the interval $\left[Q_{p}, Q_{p+1}\right]$ onto the standard interval $[-1,1]$ via

$$
\xi=\frac{2 q-Q_{p}-Q_{p+1}}{Q_{p+1}-Q_{p}}, \quad-1 \leq \xi \leq 1 .
$$

The new variable $\xi$ is local with respect to the $p$ th interval. Next, we define the three quadratic polynomials [11] $\varphi_{L}^{\left(p^{\prime}\right)}(q), \varphi_{M}^{(p)}(q)$, and $\varphi_{R}^{\left(p^{\prime}\right.}(q)$, local to $\left[Q_{p}, Q_{p+1}\right]$ :

$$
\begin{aligned}
& \varphi_{L}^{(p)}(q)=-\frac{1}{2} \xi(1-\xi), \\
& \varphi_{M}^{\left(p^{\prime}\right.}(q)=1-\xi^{2}, \\
& \varphi_{R}^{\left(p^{\prime}\right)}(q)=\frac{1}{2} \xi(1+\xi) .
\end{aligned}
$$

These local functions are depicted in Figs. $2 \mathrm{a}-2 \mathrm{c}$. They have the following properties: $\varphi_{L}^{(p)}\left(Q_{p}\right)=1, \varphi_{L}^{(p)}\left(\bar{Q}_{p}\right)$ $=\varphi_{L}^{(p)}\left(Q_{p+1}\right)=0 ; \varphi_{M}^{(p)}\left(\bar{Q}_{p}\right)=1, \varphi_{M}^{\left(p^{\prime}\right)}\left(Q_{p}\right)=\varphi_{M}^{(p)}\left(Q_{p+1}\right)=0 ;$ $\varphi_{R}^{\left(p^{\prime}\right)}\left(Q_{p+1}\right)=1, \varphi_{R}^{(p)}\left(\bar{Q}_{p}\right)=\varphi_{R}^{(p)}\left(Q_{p}\right)=0$.

The next step is to relate the local functions of Eqs. (44) to the expansion basis $\left\{u_{m}(q)\right\}$ used in Sec. II. To enforce the requirement that the wave packet vanish at $q=0$ and $q_{\max }$, we omit $\varphi_{L}^{(1)} \varphi_{R}^{(I)}$. Then the $2 I-1$ expansion functions are defined as follows:

$$
u_{2 m-1}=\left\{\begin{array}{l}
\varphi_{M}^{(m)}(q), \quad Q_{m} \leq q \leq Q_{m+1} \\
0 \text { otherwise }
\end{array}\right.
$$

where $m=1,2, \ldots, I$, and

$$
u_{2 m}=\left\{\begin{array}{l}
\varphi_{R}^{(m)}(q), \quad Q_{m} \leq q \leq Q_{m+1} \\
\varphi_{L}^{(m+1)}(q), \quad Q_{m+1} \leq q \leq Q_{m+2} \\
0 \text { otherwise }
\end{array}\right.
$$

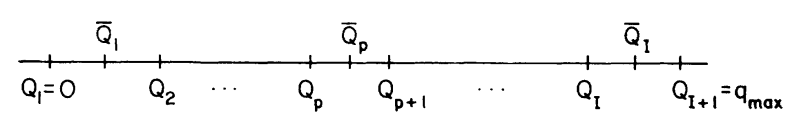

FIG. 1. A partitioning of the interval $\left[0, q_{\max }\right]$ into $I$ subintervals. The $p$ th subinterval $\left[Q_{p}, Q_{q+1}\right]$ has midpoint $\bar{Q}_{p}$, while $Q_{1}=0$ and $Q_{I+1}=q_{\max }$.

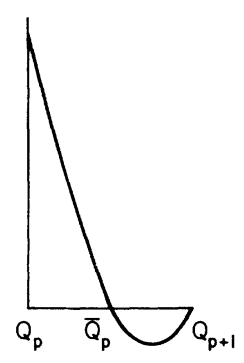

(a)

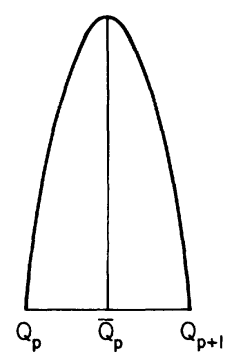

(b)

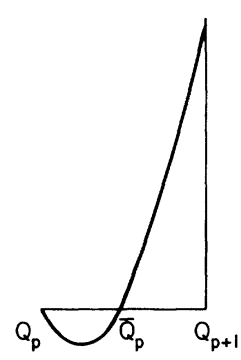

(c)
FIG. 2. Schematic representation of the three piecewise quadratics of Eq. (44) in the subinterval $\left[Q_{p}, Q_{p+1}\right]$. (a) $\varphi_{L}^{(p)}(q)$; (b) $\varphi_{M}^{(p)}(q)$; (c) $\varphi_{R}^{\left(p^{\prime}\right.}(q) ; q \varepsilon\left[Q_{p}, Q_{p+1}\right]$.

with $m=1,2, \ldots, I-1$. Thus there is one function associated with each (internal) nodal point $Q_{p}, p=2,3, \ldots, I$, and with each midpoint $\bar{Q}_{p}, p=1,2, \ldots, I$, giving a total of $2 I-1$ basis functions. Each basis function has a finite support, two subintervals for functions associated with nodal points $Q_{p}$, and one subinterval for functions corresponding to the midpoints $\bar{Q}_{p}$.

The final consideration is to collect together (and relabel) the sets of nodal points $\left\{Q_{p}\right\}$ and midpoints $\left\{\bar{Q}_{p}\right\}$ as a set of interpolation points $\left\{q_{j}\right\}_{j=1}^{j=2 I-1}$ defined via

$$
\begin{aligned}
& q_{2 p-1}=\bar{Q}_{p}, \quad p=1,2, \ldots, I \\
& q_{2 p}=Q_{p+1,} \quad p=1,2, \ldots, I-1 .
\end{aligned}
$$

Then the basis functions have the property that

$$
u_{m}\left(q_{p}\right)=\delta_{m p} \text {. }
$$

As a result, the expansion coefficients $C_{m}(t)$ and $B_{m}(t)$ can be identified as $C_{m}=\widetilde{\psi}_{q_{0}}\left(q_{m}, t\right)$, and $B_{m}=\widetilde{\phi}_{q_{0}}\left(q_{m}, t\right)$.

The set $\left\{q_{j}\right\}, j=1,2, \ldots, 2 I-1$, is also a natural choice for the collocation points, since the overlap matrix $\bar{\Delta}_{m^{\prime} m}$ ( $\equiv\left\langle q_{m}, u_{m}\right\rangle$ ) becomes the unit matrix. Other choices are possible.

\section{Specification of computational parameters}

The quantity extracted from our calculations is $\widetilde{S}_{0}(q)$, given by

$$
\widetilde{S}_{0}(q)=\frac{\widetilde{\psi}_{q_{0}}(q, T)}{\widetilde{\phi}_{q_{0}}(q, T)},
$$

where $T$ is a sampling time. We have already noted that $\widetilde{S}_{0}(q)$ is much closer to the (analytically available) exact values of $S_{0}(q)=e^{2 i \delta_{0}}$ than is the ratio $\widetilde{\psi}_{q_{0}}(q, T) / \phi_{q_{0}}(q, T)$. The reason is that the numerical time-evolution procedure introduces errors common to both $\widetilde{\psi}$ and $\widetilde{\phi}$. For $t$ small enough, $\widetilde{\phi}(t)$ and the analytically evolved $\phi(t)$ differ by a phase factor that can be characterized as a kind of numerical scattering. However, for $t$ large enough, the magnitude of the error becomes larger than unity, as we demonstrate in Sec. V by 
some comparisons of $\left|\widetilde{S}_{0}\right|^{2}$ and $\left|\widetilde{\psi}_{q_{0}} / \phi_{q_{0}}\right|^{2}$.

To extract $S_{l}(q)$ from a wave-packet calculation, one must specify the following: (i) the initial wave-packet parameters $q_{0}, r_{0}$, and $d$; (ii) the momentum cutoff $q_{\max }$; (iii) the set of nodal points $Q_{p}$ dividing the interval $\left[0, q_{\max }\right]$ into $I$ subintervals; (iv) the time step $\delta t$; and (v) a choice of method (Galerkin or collocation).

The cutoff $q_{\max }$ is determined by the extent of the momentum-space support of the wave packet and the behavior of $V\left(q, q^{\prime}\right)$. In all cases studied, $q_{\max }=8 \mathrm{fm}^{-1}$ was found adequate. Denoting by $E_{\text {max }}$ the largest eigenvalue of the Hamiltonian matrix, the stability condition for the second-order time-difference scheme is $E_{\max } \delta t<1$. For the present problem, $E_{\max } \approx q_{\max }^{2}$. Thus $\delta t=0.01 \mathrm{fm}^{2}$ was sufficient to ensure stability. In a few cases, $\delta t=0.002 \mathrm{fm}^{2}$ was used as a check, with no discernible effect on the quality of the results.

The number of mesh points as well as their placement are each an essential ingredient to achieving results. The specification of the number and distribution of nodal points $\left\{Q_{p}\right\}$ involves a number of considerations such as the width of the momentum distribution of the initial packet and the transit time. First, the mesh does not have to be evenly spaced. If we denote by $q_{L}\left(q_{H}\right)$ the lowest (highest) momentum whose probability density in a given initial packet is greater than, say, 0.005 , the mesh in the intervals $\left[0, q_{l}\right]$, and $\left[q_{H}, q_{\max }\right]$ can be taken considerably coarser than the mesh for the interval $\left[q_{L}, q_{H}\right]$, which is the effective momentum support of the wave packet.

Secondly, we observe that the coordinate-space representation $\left\langle r \mid u_{p}\right\rangle$ of the basis function $u_{p}(q)$ defined on a given partition $\left\{Q_{p}\right\}$ has negligible amplitude after a certain distance $r_{\max }$, which is roughly given by $r_{\max }=2 \pi / \Delta q$, where $\Delta q$ is the typical spacing between the momentum mesh points. Of course, this boundary is gradual rather than sharp. To obtain meaningful results, time propagation has to be stopped before the coordinate-space image of the wave packet reaches the boundary. Otherwise, high-momentum components of the wave packets will be reflected from the boundary, and the reflected (incoming) part will interfere with the slower outgoing portion. Thus the momentum discretization must be fine enough to define a time period during which the low-momentum tail for the wave packet is outside the interaction region and at the same time the highmomentum portion has not yet reached the (implicit) boundary. That is, we need a time interval $\left[t_{F}, t_{\text {max }}\right]$ during which the wave packet is free and outgoing so that the long-time lemma can be applied.

A rough estimate of mesh size can be obtained as follows. The semiclassical transit time for the free wave packet is $2 \mu r_{0} / q_{0}$. However, if the ratio $q_{0} / q_{L}$ is large, the time needed for the slow components to leave the interaction region might be much larger than the semiclassical transit time. In fact, considering the time for a free particle of momentum $q_{L}$ to move a distance of $2 r_{0}$, we obtain an estimate of $t_{F}$ as $2 \mu r_{0} / q_{L}$. Thus one should not expect to obtain accurate values of $\widetilde{S}_{0}(q)$ until $t$ is about $q_{0} / q_{L}$ times the semiclassical transit time. On the other hand, the distance traveled by the high-momentum component of the wave packet during the interval $\left[0, t_{F}\right]$ can be estimated as $q_{H} t_{F} / \mu=2 r_{0} q_{H} / q_{L}$. Therefore, $r_{\max }$ should be at least $\left[\left(2 q_{H} / q_{L}\right)-1\right] r_{0}$ if boundary reflection is to be avoided. Taking, as a rough estimate, $r_{\text {max }} \approx 2 r_{0} q_{H} / q_{L}$, the mesh spacing needed comes out as $\Delta q=2 \pi / r_{\max } \approx \pi q_{L} /\left(r_{0} q_{H}\right)$. Conversely, for a given set $\left(q_{0}, r_{0}, d\right)$ of wave-packet parameters, specification of $\Delta q$ implies $t_{F} \approx 2 \mu r_{0} / q_{L}$ and $t_{\max } \approx 2 \pi \mu /\left(q_{H} \Delta q\right)$. The condition for the validity of the long-time lemma, viz., $t_{\text {max }}>t_{F}$, then implies $q_{H} / q_{L}<\pi /\left(2 r_{0} \Delta q\right)$. Thus, for wave packets involving a large $q_{H} / q_{L}$ ratio (i.e., a small $d$ ), a very fine momentum mesh might have to be used. For a fixed value of $d$, as $q_{0}$ is lowered the ratio $q_{H} / q_{L}$ and, hence, the number of mesh points needed increases.

The above estimates ensure reflection-free wave-packet propagation, provided there are no resonances within the effective momentum support of the wave packet. However, a basis set defined on a momentum mesh fine enough to satisfy the condition $t_{\max }>t_{F}$ may not be large enough to give an accurate expansion of the wave packets. Especially, for large transit times, the oscillations of the highmomentum components (due to the $e^{-i E_{q} t}$ factor) might become severe at the later stages of the time propagation. That is, a basis expansion representation which is excellent for small $t$ can become degraded for very large $t$. The case $q_{0}=1 \mathrm{fm}$ provides an example of this. For an accurate representation of such an oscillatory function, a denser set of mesh points is required.

Adequacy of the computational parameters for a given wave packet can be measured by how well the norms of $\widetilde{\phi}_{q_{0}}(q, t)$ and $\widetilde{\psi}_{q_{0}}(q, t)$ are conserved throughout the time evolution. Even if each of these norms remains within 0.01 or 0.001 of unity for all $t$ sampled, this does not guarantee that both $\operatorname{Re} \tilde{S}_{0}(q)$ and $\operatorname{Im} \widetilde{S}_{0}(q)$ will be equally accurate for each value of $q$. In general, those $q$ closest to $q_{0}$ yield the best $\widetilde{S}_{0}(q)$, in both the Galerkin and the collocation cases. A basis-size deficiency and/or a boundary reflection will be manifested as oscillations in the computed values of $\widetilde{S}_{0}(q)$. An example of this is discussed in Sec. V.

The arguments above show that the width parameter $d$ has a direct bearing on the computational cost of a wave-packet calculation. For a given $q_{0}$, as $d$ gets smaller, the basis size (hence the computational effort) needed to achieve equivalent accuracy gets larger, but, at the same time, $S$ matrices over a larger energy range can be extracted. A reasonable compromise is to choose $d$ to give a relatively low ratio of $q_{H} / q_{L}$. In the cases studied, the value $d=2 \mathrm{fm}$, for which $q_{L} \approx q_{0}-1 \mathrm{fm}^{-1}$ and $q_{H} \approx q_{0}+1 \mathrm{fm}^{-1}$, was nearly optimal.

Calculations have been carried out for the values $q_{0}=1,2$, and $4 \mathrm{fm}^{-1}$, corresponding to energies of approximately 30,120 , and $480 \mathrm{MeV}$, respectively $\left(\mu=M_{p} / 2\right)$. For $q_{0}=4 \mathrm{fm}^{-1}$, the value $I=54$ was used, while for the two smaller $q_{0}$ the following were employed to study the dependence on $I: q_{0}=2 \mathrm{fm}^{-1}, I=55,100$, and $110 ; q_{0}=1 \mathrm{fm}^{-1}, I=150$ and 300 . Various mesh spacings were used in the computations, with most of the mesh points covering the interval $\left[q_{L}, q_{H}\right]$. We remark 
again that the same mesh spacings and time intervals must be used in calculating the free and full wave packets.

\section{RESULTS}

Although calculations have been performed for $q_{0}=1$, 2 , and $4 \mathrm{fm}^{-1}$, most of the results presented and discussed in this section are for $q_{0}=2 \mathrm{fm}^{-1}$, as this case is reasonably representative. A brief description of the $q_{0}=1$ and $4 \mathrm{fm}^{-1}$ cases is also included.

The goal of our calculations has been to produce $\widetilde{S}_{0}(q)$ 's of sufficient accuracy. In achieving this aim, we have studied how $\widetilde{S}_{0}(q)$ varies with the sampling time $T$; with the time step $\delta t$; with the number and placement of both the mesh points $\left\{q_{j}\right\}$ and the collocation points $\left\{q_{\alpha}\right\}$ (where applicable); and finally with the type of time evolution equation, viz., Galerkin or collocation. In addition to examining these points, we have also investigated the momentum- and coordinate-space behavior of the wave packet as it evolves under the action of the full Hamiltonian $\boldsymbol{H}$. Unitarity was tested by calculating $\widetilde{S}_{0}^{*}(q) \widetilde{S}_{0}(q)$. This is a more stringent measure of the stability of the methods than the computation of the norms as the packets evolve in time.

The $q_{0}=2 \mathrm{fm}^{-1}$ calculations have been carried out with $I=55,100$, and 110 . The distributions of mesh points are $0(0.1) 4(0.2) 6(0.4) 8$ for $I=55 ; \quad 0(0.05) 4(0.1) 6(0.2) 8 \quad$ for $\quad I=110, \quad$ and $0(0.05) 3.6(0.1) 4.8(0.2) 8$ for $I=100$. Here the notation $q(\Delta q) q^{\prime}$ means that the interval $\left[q, q^{\prime}\right]$ is divided into equal finite elements of length $\Delta q$. Although accurate results could be obtained as early as $T=8 \mathrm{fm}^{2}$, time propagation was continued up to $T=25 \mathrm{fm}^{2}$ in order to demonstrate the issues that come up in connection with very long time propagation. In general, changes in $I$ and $\delta T$ had no effect on $\widetilde{S}_{0}(q)$ to (at worst) the third decimal place, although other quantities such as the probability densities did show some variations, some examples of which are noted in the following. On the other hand, at the larger sampling times, $T \geq 19 \mathrm{fm}^{2}, \widetilde{S}_{0}(q)$ did show deviations from $S_{0}(q)$ at either the higher or lower values of $q$, although these deviations are much less than exhibited by the wave packets.

The value $I=100$ was used in generating the results displayed in this section. Shown will be the values of $\operatorname{Re} \widetilde{S}_{0}(q)$ and $\operatorname{Im} \widetilde{S}_{0}(q)$ as well as the momentum-space

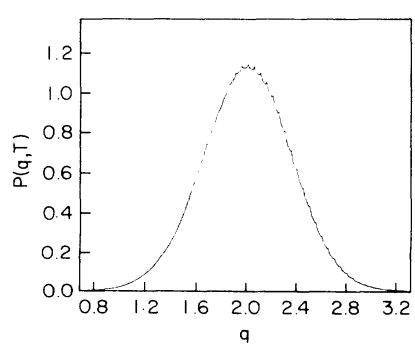

(a)

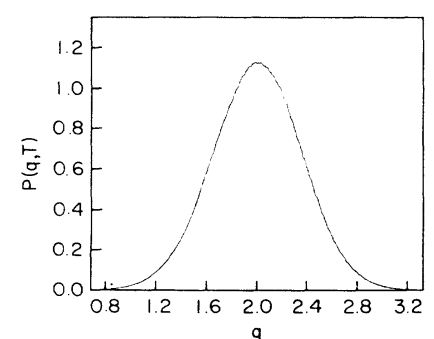

(b)
FIG. 3. Momentum-space probability distribution for $q_{0}=2$ $\mathrm{fm}^{-1}$ and $I=100$ at $T=8 \mathrm{fm}^{2}$ (a) Galerkin case; (b) collocation results.

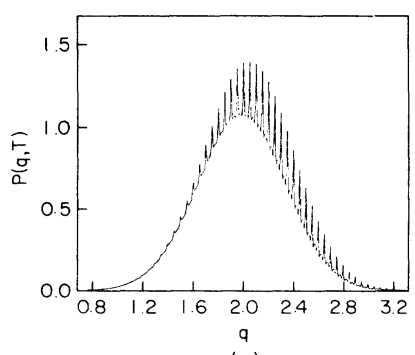

(a)

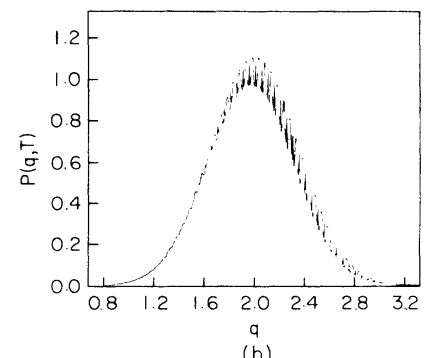

(b)
FIG. 4. Momentum-space probability distributions for $q_{0}=2$ $\mathrm{fm}^{-1}$ and $I=100$ at $T=16 \mathrm{fm}^{2}$. (a) Galerkin calculation; (b) collocation case.

and position-space probability densities, $P(q, T)$ $\left[\equiv\left|\widetilde{\psi}_{q_{0}}(q, T)\right|^{2}\right]$ and $P(r, T) \quad\left(\equiv\left|\left\langle r \mid \widetilde{\psi}_{q_{0}}\right\rangle\right|^{2}\right)$, each at selected values of $T$. $P(r, T)$ was obtained from the Fourier transform of the $\widetilde{\psi}_{q_{0}}(q, T)$.

The momentum-space probability density at $T=0$ was significantly different from zero only for $q$ in the range $1.0 \leq q \leq 3.0 \mathrm{fm}^{-1}$. We have therefore plotted $\widetilde{S}_{0}(q)$ and $P(q, T)$ only for $q$ in this same range. Note that at the upper end of this range, the semiclassical velocity is 6 $\mathrm{fm}^{-1}$, so that after $T=25 \mathrm{fm}^{2}$, that portion of the free wave packet would have moved out to a distance of 140 $\mathrm{fm}$, compared to the reflection boundary of about $120 \mathrm{fm}$. Although this value suggests that reflections could occur, none is evident in the extracted $\widetilde{S}_{0}(q)$ values, since the weight of the $q=5 \mathrm{fm}^{-1}$ component in the momentum distribution is quite low. However, there is evidence for reflection in the calculation with $I=55$, as will be discussed later on.

Figure 3 shows the momentum-space probability distributions at $T=8 \mathrm{fm}^{2}$. Their overall shapes are the same as at $T=0$, but unlike the smooth curves obtained at $T=0$, both the Galerkin and collocation curves show wiggles for $q>2 \mathrm{fm}^{-1}$. These wiggles become more pronounced and spread to smaller values of $q$ as $T$ increases, as seen in the $T=16 \mathrm{fm}^{2}$ curves (Fig. 4). The variations in height are rather more pronounced in the Galerkin case than in the collocation case. By $T=24 \mathrm{fm}^{2}$ (Fig. 5), the height variations in the Galerkin calculations have become much greater than in the $T=16 \mathrm{fm}^{2}$ case and are again significantly greater than in the corresponding $T=24 \mathrm{fm}^{2}$, collocation results.

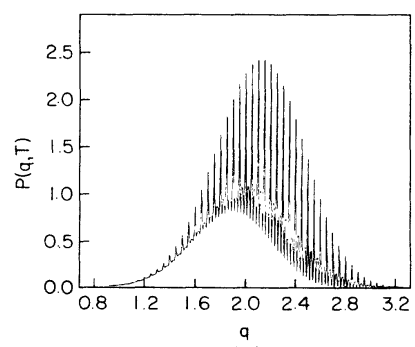

(a)

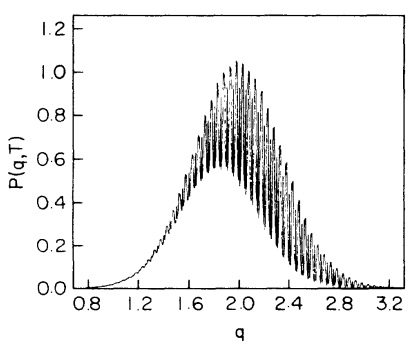

(b)
FIG. 5. Momentum-space probability distributions for $q_{0}=2$ $\mathrm{fm}^{-1}$ and $I=100$ at $T=24 \mathrm{fm}^{2}$. (a) Galerkin results; (b) collocation calculation. 
TABLE I. Comparison of $\left|\widetilde{S}_{0}\right|^{2}$ with $|\widetilde{J}|^{2}=\left|\widetilde{\psi}_{q_{0}}(q, T) / \phi_{q_{0}}(q, T)\right|^{2}$ for $q_{0}=2 \mathrm{fm}^{-1}$ and $q=2 \mathrm{fm}^{-1}$ at various sampling times $T$.

\begin{tabular}{ccc}
\hline$T\left(\mathrm{fm}^{2}\right)$ & $\left|\widetilde{S}_{0}\right|^{2}$ & $|\widetilde{J}|^{2}$ \\
\hline 6 & 1.0153 & 1.0147 \\
8 & 1.0058 & 1.0165 \\
12 & 1.0058 & 1.0968 \\
16 & 1.0061 & 1.2942 \\
20 & 1.0052 & 1.6772 \\
24 & 1.0038 & 2.3740 \\
\hline
\end{tabular}

Ideally the momentum probability density should be smooth and independent of $T$ for sufficiently large $T$, because $\left|S_{0}\right|^{2}=1$ and $\psi_{q_{0}}(q, T)=S_{0}(q) \phi_{q_{0}}(q, T)$ implies $\left|\psi_{q_{0}}(T)\right|^{2}=\left|\phi_{q_{0}}(T)\right|^{2}=\left|\phi_{q_{0}}(0)\right|^{2}$. The fact that the ratio $\widetilde{\psi}_{q_{0}}(q, T) / \widetilde{\phi}_{q_{0}}(q, T)$ does not show such oscillations indicates that essentially identical wiggles plague the numerical free wave packet and its momentum probability density. In fact, the $\left|\widetilde{\phi}_{q_{0}}(q, T)\right|^{2}$ vs $q$ plots are practically indistinguishable from those of $\left|\widetilde{\psi}_{q_{0}}(q, T)\right|^{2}$, for $T>t_{F}$. These wiggles are a reflection of the inability of the expansion basis to represent the rapid variations in $\widetilde{\psi}_{q_{0}}(q, T)$ and $\widetilde{\phi}_{q_{0}}(q, T)$ for large $T$. Nevertheless, as discussed later in more detail, the extracted $\widetilde{S}_{0}(q)$ values are quite accurate, and satisfy unitarity to a high degree. In general, $\left|\widetilde{S}_{0}\right|^{2}$ differed from unity in the third (or higher) decimal place for $T$ large enough. We also have calculated $|\widetilde{J}|^{2}$, where $\widetilde{J} \equiv \widetilde{\psi}_{q_{0}}(q, T) / \phi_{q_{0}}(q, T)$ is the ratio of the numerically propagated wave packet to the exact time-evolved free wave packet. This ratio demonstrates very convincingly the cancellation of the numerical errors common to $\bar{\psi}_{q_{0}}$ and $\widetilde{\phi}_{q_{0}}$, but which do not occur in $\phi_{q_{0}}$. Some comparisons of $\left|S_{0}\right|^{2}$ and $|\widetilde{J}|^{2}$ are given in Table I for the Galerkin case with $I=110$. We see that the error in $|\widetilde{J}|^{2}$ increases with increasing $q$ and $T$ (it is smaller at larger $T$ for $q=1 \mathrm{fm}^{-1}$ than in any of the entries in Table $I$ at the same $T$ ), and

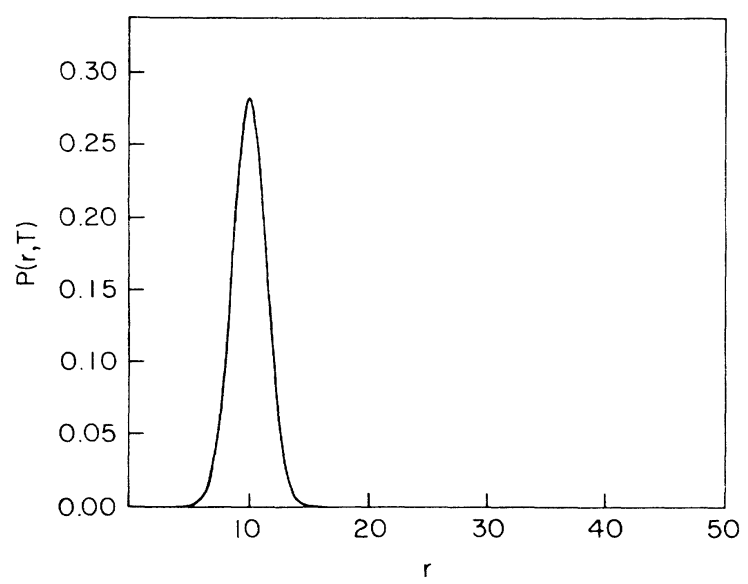

FIG. 6. Position-space probability distribution for $I=100$ at $T=0$ (Galerkin case).

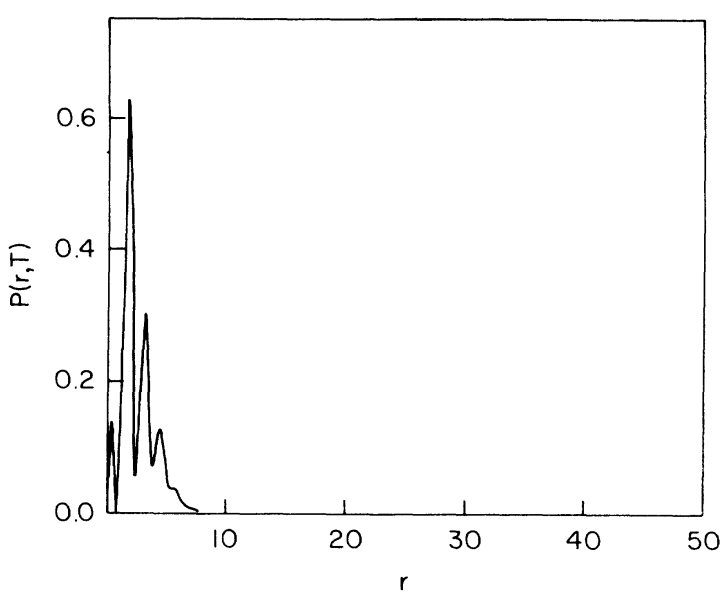

FIG. 7. Position-space probability distribution for $I=100$ at $T=2 \mathrm{fm}^{2}$ (Galerkin case).

that only at the smaller $T$, where numerical propagation errors are expected to be small, is $|\widetilde{J}|$ reasonably close to unity. The need to work with $\widetilde{\phi}_{q_{0}}$ is evident from this table.

In Figs. 6-11, we display $P(r, T)$, the position-space probability density for the Galerkin case. The $T=0$ curve of Fig. 6 is a standard Gaussian shape; the piecewise quadratic approximation is excellent. At $T=2 \mathrm{fm}^{2}$ (Fig. 7) and $T=4 \mathrm{fm}^{2}$ (Fig. 8), $P(r, T)$ clearly shows oscillations; these occur because the interaction is no longer negligible. In neither case has the center of the packet $r_{c}$ returned to its initial position of $10 \mathrm{fm}$. By $T=8 \mathrm{fm}^{2}$ (Fig. 9), $r_{c}$ is about $23 \mathrm{fm}$ and the packet has become smooth and has spread out considerably. From this latter time until $T=12 \mathrm{fm}^{2}$, the shape of $P(r, T)$ is smooth and the packet behaves as expected. Then at $T=13 \mathrm{fm}^{2}$, a slight wiggle occurs at the large $r$ side of the packet. By $T=16 \mathrm{fm}^{2}$, this phenomenon has spread over almost all of the packet, being most pronounced near and to the right of the peak. Finally, at $T=24 \mathrm{fm}^{2}$ (Fig. 11), the entire $P(r, T)$ curve shows these oscillations

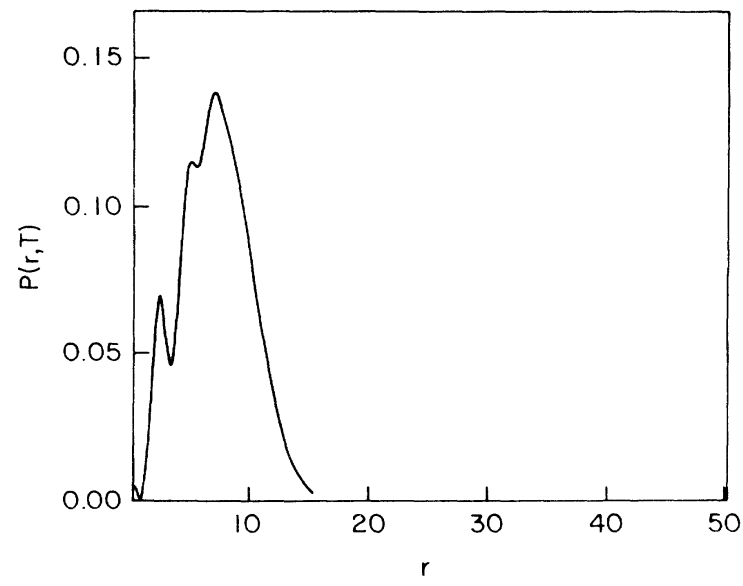

FIG. 8. Position-space probability distribution for $I=100$ at $T=4 \mathrm{fm}^{2}$ (Galerkin case). 


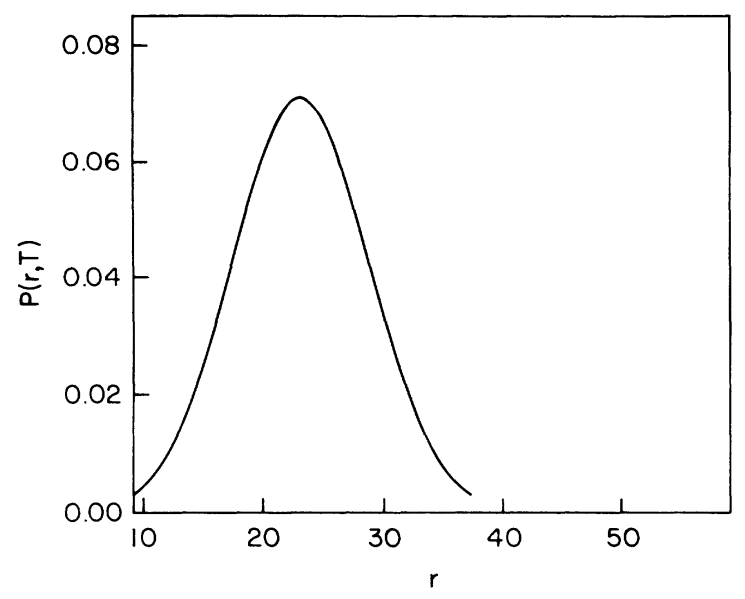

FIG. 9. Position-space probability distribution for $I=100$ at $T=8 \mathrm{fm}^{2}$ (Galerkin case).

everywhere, with those of largest amplitude occurring at the largest values of $r$ at which $P(r, T)$ was determined, viz., $r \simeq 127 \mathrm{fm}$. Although this latter distance is greater than the boundary of $\sim 120 \mathrm{fm}$, these oscillations are not a manifestation of interference due to reflection. Supporting this conclusion are the facts that (i) $r_{c}$ at $T=24$ $\mathrm{fm}^{2}$ is greater than $r_{c}$ at $t=23 \mathrm{fm}^{2}$ and (ii) the overall shape of $P(r, T)$ for $r>r_{c}$ is one in which the magnitude decreases as $r$ increases.

These figures raise intriguing questions. For example, for $T=8 \mathrm{fm}^{2}$, the smoothness of both $P(q, T)$ and $P(r, T)$, plus the fact that $r_{c}$ is about $23 \mathrm{fm}$, suggests that the particles are well separated and that the packets are propagating under $H_{0}$. But, since $T$ is only $8 \mathrm{fm}^{2}$ (a number seemingly not asymptotic), can accurate values of $\widetilde{S}_{0}(q)$ be obtained from implementation of the long-time lemma at this apparently small value of $T$ ? Furthermore, as $T$ increases to larger values which might justify use of the long-time lemma, will the oscillations in the probability densities prevent the extraction of $\widetilde{S}_{0}(q)$ 's of sufficient ac-

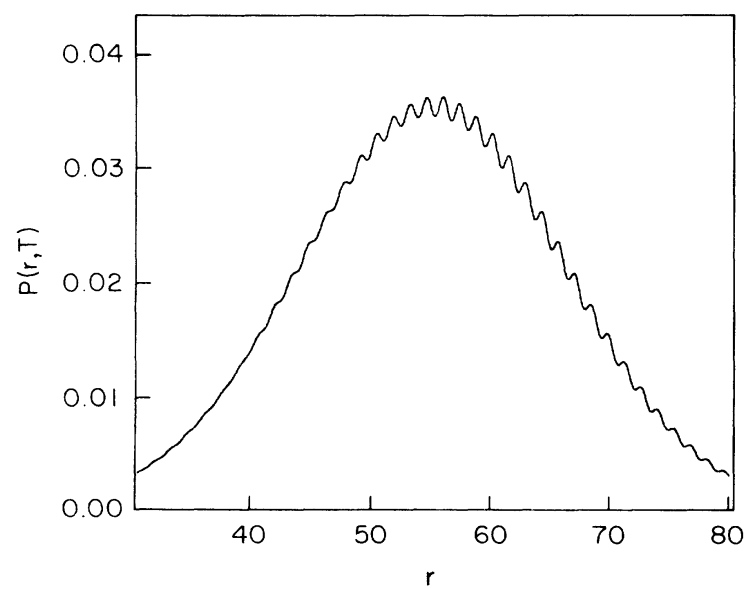

FIG. 10. Position-space probability distribution for $I=100$ at $T=16 \mathrm{fm}^{2}$ (Galerkin case).

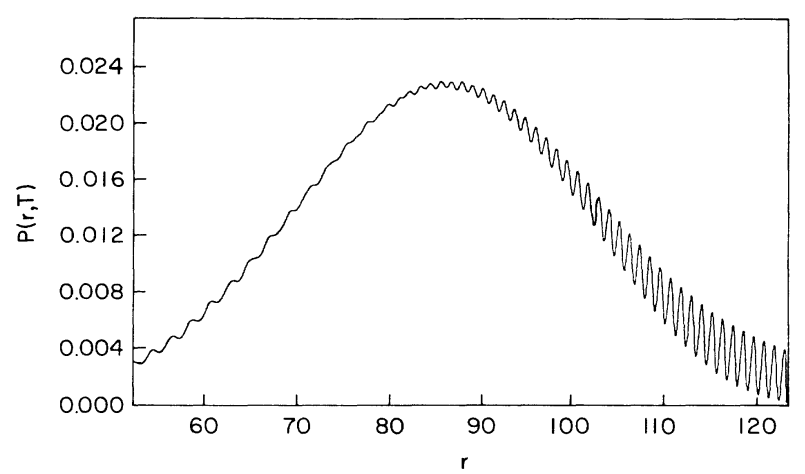

FIG. 11. Position-space probability distribution for $I=100$ at $T=24 \mathrm{fm}^{2}$ (Galerkin case).

curacy? The answers to these questions are yes and no, respectively, as we demonstrate in the following.

As noted earlier, values of $\operatorname{Re} \widetilde{S}_{0}(q)$ and $\operatorname{Im} \widetilde{S}_{0}(q)$ have been extracted for $q$ in the range $1.0 \leq q \leq 3.0 \mathrm{fm}^{-1}$. At $T=6 \mathrm{fm}^{2}$, both the Galerkin and the collocation procedures produce fairly accurate values of $\widetilde{\boldsymbol{S}}_{0}(q)$ for $q$ greater than about $1.6 \mathrm{fm}$. This is an unexpectedly low value of $T$, yet one for which the long-time lemma is reliable, although not over the full range of $q$. At $T=8 \mathrm{fm}^{2}$ the interval of reliability has become $\left[1.3 \mathrm{fm}^{-1}, 3.0\right.$ $\mathrm{fm}^{-1}$, with the collocation results slightly more accurate than those from the Galerkin analysis. The less accurate Galerkin values of $\widetilde{S}_{0}(q)$ for $T=8 \mathrm{fm}^{2}$ are shown in Fig. 12. Except for the most slowly moving (lower $q$ ) positions of the wave packet, the long-time lemma is evidently functioning quite reliably. The crucial point is that it is the product $q^{2} T$ rather than $T$ itself which is the better measure of asymptotia.

Jumping to $T=16 \mathrm{fm}^{2}$, for which the wiggles in $P(q, T)$ extend over much of the $q$ interval, the overall agreement between $\widetilde{S}_{0}(q)$ and $S_{0}(q)$ is excellent (as it is also at $T=13,14$, and $15 \mathrm{fm}^{2}$ ), the biggest deviations being seen at low $q$ for $\operatorname{Im} \widetilde{S}_{0}(q)$, where they are a few percent in the Galerkin case, results for which are shown in Fig. 13. This case is especially important, since it shows that by forming $\widetilde{\psi}_{q_{0}}(q, T) / \widetilde{\phi}_{q_{0}}(q, T)$, the oscillations in


FIG. 12. Comparison of exact ( - ) and Galerkin-based wave-packet ( . - . - ) values of the $l=0 S$-matrix elements for $q_{0}=2 \mathrm{fm}^{-1}$ and $I=100$ at $T=8 \mathrm{fm}^{2}$. (a) Real parts; (b) imaginary parts. 

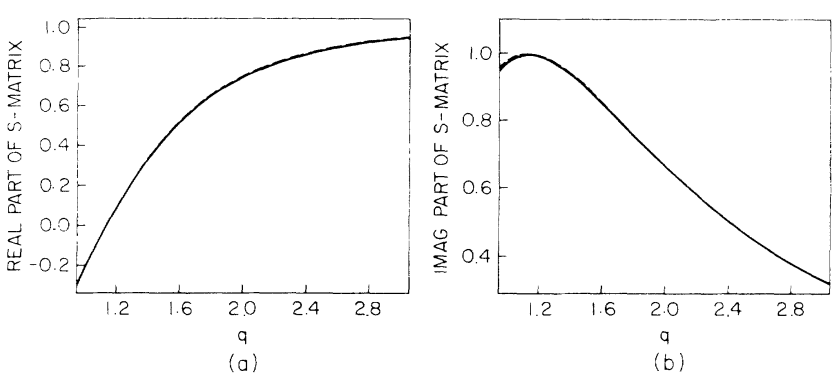

FIG. 13. Comparison of exact ( -$)$ and Galerkin-based wave-packet ( . . . . ) values of the $l=0 S$-matrix elements for $q_{0}=2 \mathrm{fm}^{-1}$ and $I=100$ at $T=16 \mathrm{fm}^{2}$. (a) Real parts; (b) imaginary parts.

$\widetilde{\psi}_{q_{0}}(q, T)$ are indeed canceled by those in the numerically propagated free wave packet, as previously claimed.

The accuracy of $\widetilde{S}_{0}(q)$ continues to range from very good (1-2\% error) to excellent over the whole interval $\left[1.0 \mathrm{fm}^{-1}, 3.0 \mathrm{fm}^{-1}\right]$ up to $T=20 \mathrm{fm}^{2}$, with the collocation values being slightly the better ones. At $T=21 \mathrm{fm}^{2}$, this changes, and the collocation values of $\widetilde{S}_{0}(q)$ show, at the largest $q$, deviations whose size is about equal to those from the Galerkin calculations. This behavior becomes more pronounced as $T$ increases; Fig. 14 gives a comparison between the Galerkin and collocation results for $T=24 \mathrm{fm}^{2}$, where the errors in the collocation-derived $\widetilde{S}_{0}(q)$ values for $q \geq 2.4 \mathrm{fm}^{-1}$ are striking. Due to the $q^{2} T$ factor, the deficiency of the basis size in representing a highly oscillatory function manifests itself first in the high-momentum tail of the wave packet. Evidently, the



(a)
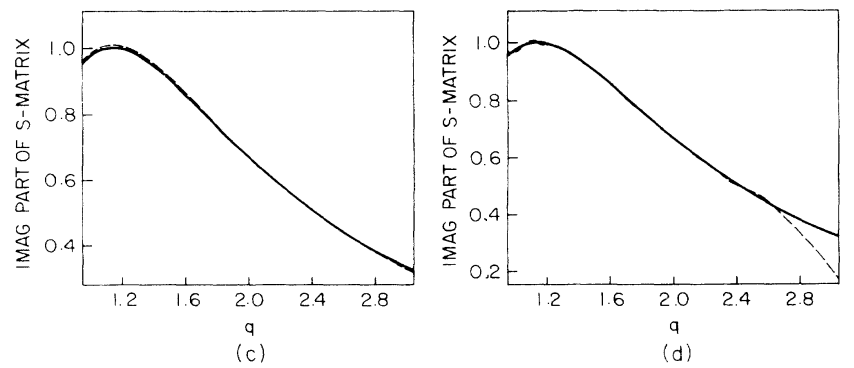

FIG. 14. Comparison of exact (-) and both the Galerkin- and collocation-based wave-packet ( . . . - ) values of the $l=0 S$-matrix elements for $I=100$ at $T=24 \mathrm{fm}^{2}$. (a) Real parts (Galerkin); (b) real parts (collocation); (c) imaginary parts (Galerkin); (d) imaginary parts (collocation).
Galerkin procedure is more efficient in minimizing the error term $|\mathscr{E}\rangle$ [of Eq. (26)] than the collocation procedure.

We close this subsection by noting that the results for $I=55$ are noticeably poorer than those for $I=100$ or 110. Since the same momentum interval is spanned for each of the preceding values of $I$, it is clear that $\Delta q$ for $I=55$ is, on average, about twice that for $I=100$ or 110 . Hence fewer interpolates are being used to span larger mesh intervals. Not only does this mean a less accurate representation of the solution when $I=55$, it also leads to a spatial domain of about $60 \mathrm{fm}$, half the size of the $I=100$ and 110 cases. The behavior of $r_{c}(T)$ suggests that interference due to the reflection of high-momentum components begins to occur after $T \approx 14 \mathrm{fm}^{2}$. From $T=6$ to $14, r_{c}$ increases linearly with $T$, in accordance with the semiclassical view of an outgoing free wave packet. Then the packet slows down and $r_{c}$ reaches a maximum value of about $57 \mathrm{fm}$ at $T=17 \mathrm{fm}^{2}$, after which $r_{c}$ starts to decrease. In fact, by $T=25 \mathrm{fm}^{2}$, the center of the packet recedes to about $36 \mathrm{fm}$.

Interference effects due to reflection also show up in $\widetilde{\boldsymbol{S}}(q)$, although at somewhat larger times than suggested by the behavior of $r_{c}$. In fact, $\widetilde{S}(q)$ vs $q$ curves do not exhibit any discernible effect of reflection until after $T=20$ $\mathrm{fm}^{2}$. This is quite remarkable in view of the fact that $r_{c}=53.9 \mathrm{fm}$ at $T=20 \mathrm{fm}^{-2}$. Figure 15 shows $\operatorname{Re} \widetilde{S}_{0}(q)$ and $\operatorname{Im} \widetilde{S}_{0}(q)$ for $T=25 \mathrm{fm}^{2}$, a time at which the packet is once again free, but incoming. The interference phenomenon occurs for $q>2.1 \mathrm{fm}^{-1}$, and is especially strong in $\operatorname{Im} \widetilde{S}_{0}(q)$ for $q=2.4 \mathrm{fm}^{2}$. Note that the discrepancy between $\operatorname{Im} \widetilde{S}_{0}(q)$ and $\operatorname{Im} S_{0}(q)$ for $q \leq 2.1$ $\mathrm{fm}^{-1}$ is a characteristic of the calculations for all $T$, not just the largest values. These discrepancies arise from the inability of the basis for $I=56$ to accurately represent the evolving wave packet. Use of a sufficiently large $I$ is essential if the interference and inaccuracy problems are to be avoided. This is especially important in the threeparticle case.

For $q_{0}=1 \mathrm{fm}^{-1}$ two Galerkin calculations were performed, one for which $I=150$, the other having $I=300$. In order to reduce the $q_{H} / q_{L}$ ratio, the width parameter $d$ was taken as $2.7 \mathrm{fm}$. Thus the range of $q$ for which sufficiently accurate $\widetilde{S}_{0}(q)$ is obtained is narrower for $q_{0}=1 \mathrm{fm}^{-1}$ than in the $q_{0}=2 \mathrm{fm}^{-1}$ case. With $q_{0}=1.0$ $\mathrm{fm}^{-1}$ and $d=2.7 \mathrm{fm}$, the range for which the error is $2 \%$
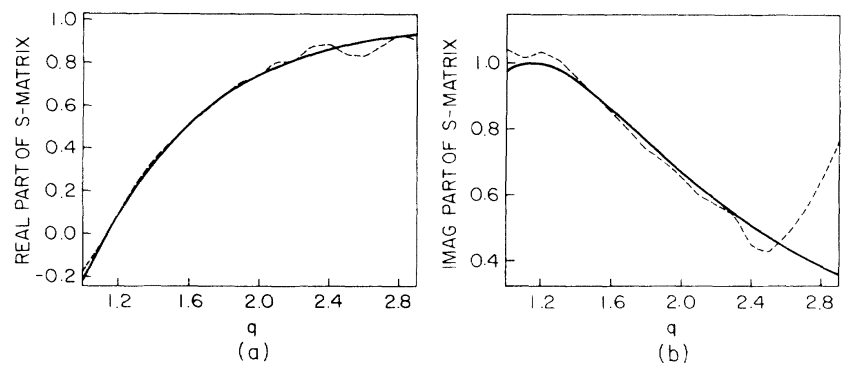

FIG. 15. Comparison of exact ( $\_$) and Galerkin-based wave-packet ( . . . - ) values of the $l=0 S$-matrix elements for $q_{0}=2 \mathrm{fm}^{-1}$ and $I=55$ at $T=25 \mathrm{fm}^{2}$. (a) Real parts; (b) imaginary parts. 
or less in both $\operatorname{Re} \widetilde{S}_{0}(q)$ and $\operatorname{Im} \widetilde{S}_{0}(q)$ is $0.6 \leq q \leq 1.8 \mathrm{fm}^{-1}$ for both $I=150$ and 300 . This accuracy is typically obtained for $T \geq 32 \mathrm{fm}^{2}$. This same behavior was found when collocation was used $(I=150)$. No improvement in the accuracy of $\widetilde{S}_{0}(q)$ was obtained when going from $I=150$ to 300 , even though the wave-packet norm becomes more accurate.

For $q_{0}=4 \mathrm{fm}^{-1}$, the smallest $q$ interval was $0.1 \mathrm{fm}^{-1}$, implying a reflection boundary at about $60 \mathrm{fm}$. Time propagation was continued until $T=10 \mathrm{fm}^{2}$, a value larger than the estimated $t_{\max }$ for the present case. In fact, reflection phenomena manifests itself in $r_{c}(T)$ starting at about $T=8 \mathrm{fm}^{2}$. However, it does not exhibit a pronounced effect on the accuracy of $\widetilde{S}_{0}(q)$ for $q$ in the range $\left[q_{0}-1 \mathrm{fm}^{-1}, q_{0}+1 \mathrm{fm}^{-1}\right]$ until $T=10 \mathrm{fm}^{2}$. $\operatorname{Im} \widetilde{S}_{0}(q)$ was extremely accurate for $T=3-8 \mathrm{fm}^{2}$ over the entire range of $q$ and lost a little accuracy for $q>4 \mathrm{fm}^{-1}$ at $T=9 \mathrm{fm}^{2}$ and lost slightly more accuracy for $q \geq 3.6$ $\mathrm{fm}^{-1}$ at $T=10 \mathrm{fm}^{2}$. $\operatorname{Re} \widetilde{S}_{0}(q)$ was most accurate over the full range of $q$ at $T=3 \mathrm{fm}^{-2}$ and became less accurate (the error is approximately equal to a few percent) with increasing $T$. This was not sufficient to cause $\left|S_{0}(q)\right|^{2}$ to be less accurate than about $99 \%$. Overall, both $\operatorname{Re} \widetilde{S}_{0}(q)$ and $\operatorname{Im} \widetilde{S}_{0}(q)$ were correct to at least two decimal places for $T>3 \mathrm{fm}^{2}$ and $q$ in the range $\left[q_{0}-1 \mathrm{fm}^{-1}, q_{0}+1\right.$ $\left.\mathrm{fm}^{-1}\right]$.

\section{CONCLUDING REMARKS}

It has been shown that the long-time lemma is a computationally viable method for extracting sharp-energy $S$-matrix elements from a time-dependent wave-packet description of two-body scattering. For each of the three central momenta $q_{0}$ considered, viz., 1,2 , and $4 \mathrm{fm}^{-1}$, accurate values of $S_{0}(q)$ have been obtained for $q$ within roughly $1 \mathrm{fm}^{-1}$ of $q_{0}$, with $q \simeq 0.6 \mathrm{fm}^{-1}$ being the smallest momentum for which the method has been successful. Very likely smaller $q\left(q_{0}\right)$ could be used, but that would mean a much larger number of mesh points.

The key elements of the calculation are the use of numerically propagated free wave packets and the momentum-space formulation of the problem. The latter choice requires that one deals with potentials expressed as integral operators, for which the present case of a se- parable potential is an important simplification. In the more general case of potentials which are local in position space, the integral-operator, momentum-space form can be circumvented by performing the relevant integrals in coordinate space and then transforming. Such Fourier transformations would presumably be an essential part of any calculation, just as they are in the present case, since they relate position and momentum-space wave packets.

The use of numerically propagated free wave packets compensates for the inaccuracies that arise due to the numerical time evolution: the momentum densities show wiggles, in some cases quite large, that are characteristic of decreased accuracy, yet the $S$-matrix elements, which are the ratios of the numerically propagated full and free wave packets, are remarkably stable and accurate. We also note that the constant support of the momentumspace wave packets allows for very long time propagation. This would lead to a very large coordinate-space domain, if the calculation were to be performed in position space, with its attendant diminishing of the wave packets via spreading.

The purpose of these calculations has been to demonstrate both the feasibility and accuracy of the method, as well as to explore some of the ranges of validity of the various parameters. The method is an essential ingredient in the three-particle computations we have performed, but is seen to be a useful and interesting alternative to the more standard time-dependent ones involving either position-space and boundary conditions or momentum-space and singularity analysis.

\section{ACKNOWLEDGMENTS}

One of us (Z.C.K.) gratefully acknowledges support from the Turkish National Research Council (TUBITAK), through Grant No. TBAG-1088. It is also a pleasure to acknowledge support from the U.S. Department of Energy via research Grant No. DE-FG0287ER40334 and computing time on the CRAY computers made available to us at the National Energy Research Supercomputing Center at Lawrence Livermore National Laboratory. We are grateful to Bilkent University and Brown University for their support and contributions to our computing efforts.
[1] J. R. Taylor, Scattering Theory (Wiley, New York, 1972); R. G. Newton, Scattering Theory of Waves and Particles, 2nd ed. (Springer-Verlag, New York, 1982).

[2] For applications to data analysis, see, e.g., G. R. Satchler, Direct Nuclear Reactions (Oxford University Press, New York, 1983).

[3] Recent developments in TDWP methodology, as well as references to earlier work, can be found in the Special Issue of Comput. Phys. Commun. 63 (1-3) (1991).

[4] For a review of pre-1987 TDWP methods and calculations, see V. Mohan and N. Sathamurty, Comput. Phys. Rep. 7, 214 (1988). A more recent review is given by $\mathbf{H}$. Kröger, Phys. Rep. 210, 45 (1992).
[5] Z. C. Kuruoğlu and F. S. Levin, Phys. Rev. Lett. 64, 1701 (1990); Z. C. Kuruoğlu, Phys. Rev. A 42, 6314 (1990).

[6] D. Neuhauser, R. Judson, and A. Perski, Chem. Phys. Lett. 181, 151 (1991); R. S. Judson, D. J. Kouri, D. Neuhauser, and M. Baer, Phys. Rev. A 42, 351 (1990); D. Neuhauser and M. Baer, J. Chem. Phys. 91, 4651 (1989).

[7] J. Z. H. Zhang, J. Chem. Phys. 92, 324 (1990); S. Das and D. J. Tannor, ibid. 92, 3403 (1990).

[8] J. Holz and W. Glöckle, Phys. Rev. C 37, 1386 (1988); D. K. Hoffman, O. Sharafeddin, R. S. Judson, and D. J. Kouri, J. Chem. Phys. 92, 4167 (1990); O. A. Sharafeddin, R. S. Judson, D. J. Kouri, and D. K. Hoffman, ibid. 93, 5580 (1990). 
[9] A. Askar and A. S. Cakmak, J. Chem. Phys. 68, 2794 (1978).

[10] See, e.g., D. Kosloff and R. Kosloff, J. Comput. Phys. 52, 35 (1983); M. D. Feit, J. A. Fleck, and A. Steiger, ibid. 47, 412 (1982); T. J. Park and J. C. Light, J. Chem. Phys. 85,
$5870(1986)$.

[11] P. M. Prenter, Splines and Variational Methods (Wiley, New York, 1975); C. A. J. Fletcher, Computational Galerkin Methods (Springer, New York, 1984).

[12] Y. Yamaguchi, Phys. Rev. 95, 1628 (1954). 